\title{
Cancer Testing for Women begins at 21
}

\author{
Rahul Hajare* \\ Indian Council of Medical Research, Vinayaka Mission University, India \\ *Corresponding author: Rahul Hajare, Indian Council of Medical Research, Ph.D Scholar Batch-2007-12 Vinayaka Mission University India, New Delhi, \\ India
}

Submission: 悳 March 26, 2018; Published: 眥 April 26, 2018

\section{Statement of Purpose}

When one reads about the challenges facing humanity this century, it becomes clear we must do more with less. A growing population must be provided with welfare while using fewer resources, particularly water, land (working surface) and energy. An aging population, chronic degenerative diseases, addictions, healthcare costs and the integration of health systems all entail challenges in the health department. However, we must not forget the presence of old and new infections that "threaten" to become pandemics. Here I will address the subject of global epidemiology and, in particular, the risk of what can be termed "global" pandemics, as was the case, a century ago, of Spanish influenza. By conservative estimates, this killed 50 million people (some sources estimate double the number of deaths).

Cancer, also called malignancy, is an abnormal growth of cells. There are more than 100 types of cancer, including breast cancer, skin cancer, lung cancer, colon cancer, prostate cancer, and lymphoma. Symptoms vary depending on the type. Cancer treatment may include chemotherapy, radiation, and/or surgery. The earlier cancer is diagnosed and treated, the better the chance of its being cured. Some types of cancer-such as those of the skin, breast, mouth, testicles, prostate, and rectum-may be detected by routine self-exam or other screening measures before the symptoms become serious. Most cases of cancer are detected and diagnosed after a tumor can be felt or when other symptoms develop. In a few cases, cancer is diagnosed incidentally as a result of evaluating or treating other medical conditions.

Symptoms women has been accepted as cause of symptoms should not Ignore. Women's bodies are always changing. Sometimes changes that seem normal can be signs of cancer, though. The key is to pay attention to body so you can notice when something has different. Cancer in women can present in many ways, but awareness levels of the key signs are very low and anatomical cause has not pay attention towards the physics of women and new symptoms indicate something has changed in women body.

Cancer diagnosis begins with a thorough physical exam and a complete medical history. Laboratory studies of blood, urine, and stool can detect abnormalities that may indicate cancer. When a tumor is suspected, imaging tests such as X-rays, computed tomography (CT), magnetic resonance imaging (MRI), ultrasound, and fiber-optic endoscopy examinations help doctors determine the cancer's location and size. To confirm the diagnosis of most cancers, a biopsy needs to be performed in which a tissue sample is removed from the suspected tumor and studied under a microscope to check for cancer cells. Preventive health care for women should never be ignored. Breast, Lung, Colorectal, Cervical and Stomach. These 5 cancers are the top killers for women, according to World Health Organization. Women are the centre pillars of all families. Should the burden of sickness fall on her, in a family, it can alter the entire equation within a household-physically, emotionally and financially. Over the years the causes of mortality and morbidity have changed from infective diseases like tuberculosis to chronic diseases like heart disease, diabetes and cancer. These diseases can be easily prevented if one understands the risk factors. If the risk factors are managed these diseases can be easily controlled. Preventive health screening for women is simple and non-invasive. These include tests for general wellbeing and specific tests for cancers. A complete blood count, along with liver function profile and kidney function profile, is required to know how the various systems in the body work. Lipid profile is very essential to estimate the levels of good and bad cholesterol. A high level of cholesterol will indicate a higher possibility of atherosclerosis and blockage of the vessels. In addition to these, an estimation of thyroid hormone is also essential, as it plays a role in the functioning of all the cells and tissues in the body. Women should begin cervical cancer screening at the age of 21. Between the ages of 21 and 29 they should do a pap test every three years. Women should also screen themselves for cancers like cervical and breast. There are two types of tests used for cervical cancer. The pap test finds early pre-cancerous changes in the cells. These can be easily treated. Regular pap screening can also find cervical cancer early and hence the treatment is better and more effective [1-5].

The HPV (human papilloma virus) infection is a well-established cause of cervical cancer. Between the ages of 30 and 65 they should do both a pap test and an HPV test every five years. Women over the age of 65, who have had regular screenings with normal results, 
should not screen themselves for cervical cancer. Those who have had their uterus and cervix removed, and have no history of cervical cancer or pre-cancer, should also not have themselves screened. Those who have taken HPV vaccination should still follow the screening recommendations for their age group. The other most common cancer is breast cancer. It is strongly recommended that women with an average risk of breast cancer should undergo regular mammography starting at the age 40 . Those who are between the age of 40 to 54 should be screened annually. Women of 55 and older can have biennial screening or can continue screening annually. In addition to these, tests for tuberculosis are important. A chest x-ray and a Mantoux skin test (a tool for screening for tuberculosis) also needs to be done at least once in two years. Angeli Misra is the cofounder of Lifeline Laboratory, which has labs across the Delhi and the NCR.

\section{Acknowledgement}

This research work is in completed under the supervision and guidance Renowned Laboratory Scientist, Respected Dr. Ramesh S.
Paranjape Retired Director \& Scientist 'G' National AIDS Research Institute, India. I express my deep gratitude towards Respected Sir for motivation and being great knowledge source for this work.

\section{References}

1. Paranjape R, Hajare R (2018) Common warts discharge among married male may be a critical determinant of immune dysfunction direct capture skin cancer. Clin Exp Dermatol Ther CEDT-147. DOI:10.29011/25758268/S1-047

2. Rahul Hajare (2017) The Aggression is an early cause of cancer. A narrative review of classical to modern scientific literature. Chronicle of Medicine and Surgery 1.2 (2017): 67-68.

3. Hajare R (2018) The Curve penis may hold secret to cause of cancer. a profile of primary level attending review treatment. J Nanomed Nanosci JNAN-143. DOI: 10.29011/JNAN-141. 100043.

4. Rahul Hajare (2018) Safe sex: The true principal health?" Medical Research and Clinical Case Reports 1.2: 7981.

5. Hajare RA (2009) Breast cancer and Indole -3-carbinol: Controversy of estrogen level and enzyme Elastase. Research Journal of Pharmacy and Technology, pp. 439-440. (c) Creative Commons Attribution 4.0 International License

For possible submissions Click Here

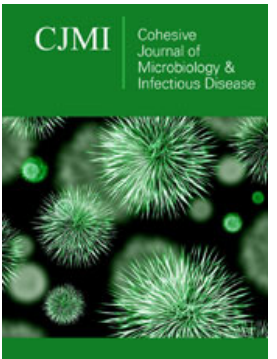

Approaches in Poultry, Dairy \& Veterinary Sciences

\section{Benefits of Publishing with us}

- High-level peer review and editorial services

- Freely accessible online immediately upon publication

- Authors retain the copyright to their work

- Licensing it under a Creative Commons license

- Visibility through different online platforms 